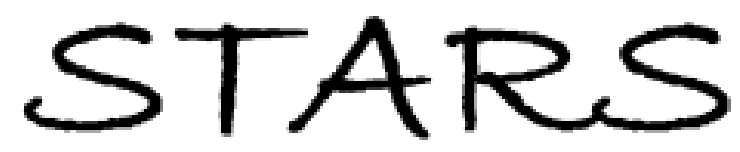

University of Central Florida

STARS

$1-1-2008$

\title{
Simultaneous excitation of fast and slow surface plasmon polaritons in a high dielectric contrast system
}

Grady Webb-Wood

University of Central Florida

Pieter G. Kik

University of Central Florida

Find similar works at: https://stars.library.ucf.edu/facultybib2000

University of Central Florida Libraries http://library.ucf.edu

This Article is brought to you for free and open access by the Faculty Bibliography at STARS. It has been accepted for inclusion in Faculty Bibliography 2000 s by an authorized administrator of STARS. For more information, please contact STARS@ucf.edu.

\section{Recommended Citation}

Webb-Wood, Grady and Kik, Pieter G., "Simultaneous excitation of fast and slow surface plasmon polaritons in a high dielectric contrast system" (2008). Faculty Bibliography 2000s. 1131.

https://stars.library.ucf.edu/facultybib2000/1131

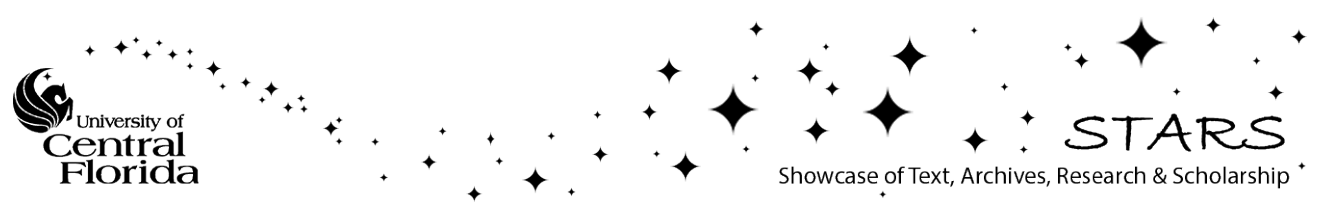




\section{Simultaneous excitation of fast and slow surface plasmon polaritons in a high dielectric contrast system}

Cite as: Appl. Phys. Lett. 92, 133101 (2008); https://doi.org/10.1063/1.2898210

Submitted: 29 October 2007 . Accepted: 10 February 2008 . Published Online: 31 March 2008

Grady Webb-Wood, and Pieter G. Kik

\section{ARTICLES YOU MAY BE INTERESTED IN}

Optimization of the slow-mode plasmon polariton in light-emitting tunnel junctions Journal of Applied Physics 78, 5522 (1995); https://doi.org/10.1063/1.359671

Near-field optical characterization of surface-plasmon-mediated light emission from electrically biased metal-insulator-semiconductor tunnel junctions

Applied Physics Letters 92, 103123 (2008); https://doi.org/10.1063/1.2898201

The slow-mode surface plasmon in planar metal-oxide-metal tunnel junctions Journal of Applied Physics 65, 1133 (1989); https://doi.org/10.1063/1.343051

Applied Physics Letters

Mid-IR and THz frequency combs special collection 


\title{
Simultaneous excitation of fast and slow surface plasmon polaritons in a high dielectric contrast system
}

\author{
Grady Webb-Wood ${ }^{\text {a) }}$ and Pieter G. Kik ${ }^{\text {b) }}$ \\ CREOL, The College of Optics and Photonics, University of Central Florida, 4000 Central Florida \\ Boulevard, Orlando, Florida 32816, USA
}

(Received 29 October 2007; accepted 10 February 2008; published online 31 March 2008)

\begin{abstract}
Surface plasmon polaritons propagating in a high dielectric contrast system are investigated numerically. Using frequency domain simulations, we show that a three layer system consisting of air-silicon (7 nm)-silver supports two different modes at the Ag-Si interface: a fast mode, which exhibits normal dispersion, and a slow mode, which exhibits anomalous dispersion. Near the Ag-Si surface plasmon polariton resonance frequency, surface waves with a wavelength of $25 \mathrm{~nm}$ are observed at a vacuum wavelength of $595 \mathrm{~nm}$, equivalent to $\lambda_{f} / 24$. The results show the possibility of exciting surface waves with extreme ultraviolet wavelengths using visible frequencies. (C) 2008 American Institute of Physics. [DOI: 10.1063/1.2898210]
\end{abstract}

Surface plasmon polaritons (SPPs) that exhibit anomalously slow phase velocities and correspondingly ultra-short wavelengths have been known to exist in metal-dielectric multilayer films since the work of Lambe and McCarthy ${ }^{1}$ on metal-insulator-metal (MIM) tunnel junctions in 1976. Subsequent theoretical ${ }^{2-4}$ and experimental ${ }^{5,6}$ work verified that slow SPP modes play a critical role in light emission from tunnel junctions, despite high losses. Recently, it has been shown that a thin, high index dielectric layer on a metal substrate with a low index cover layer can also support two different kinds of SPPs: ${ }^{7,8}$ one exhibiting positive group velocity and a relatively high phase velocity called the fast mode, and a second mode exhibiting negative group velocity and a relatively small phase velocity called the slow mode. In this kind of high-dielectric-contrast surface plasmon system, unlike the slow SPPs found in MIM structures, both the fast and slow modes have significant field amplitude in the low index surroundings, possibly allowing for direct, in situ detection of the slow modes and exploiting them for high density optical data storage, near-field microscopy, nanolithography, and surface plasmon-based sensing applications.

Though the dispersion of slow SPP modes in high dielectric contrast structures has been described for both lossless ${ }^{7}$ and lossy ${ }^{8}$ metals, no studies of the optical excitation of these modes have yet been completed. Here, we employ a finite integral, frequency domain electromagnetic solver ${ }^{9}$ to model the simultaneous excitation of slow and fast SPPs in an air-silicon-silver system via a subwavelength slit in the metal film. The simulation geometry consists of a $200 \mathrm{~nm}$ layer of silver, ${ }^{10}$ a $7 \mathrm{~nm}$ silicon ${ }^{11}$ layer, a $493 \mathrm{~nm}$ air region above the silicon, and a $60 \mathrm{~nm}$ air spacer layer below the silver layer to enable plane wave excitation of the slit. This $7 \mathrm{~nm}$ silicon thickness was chosen based on theoretical work showing that this thickness results in the maximum field propagation length of $174 \mathrm{~nm}$ for the slow mode. ${ }^{12}$ The $200 \mathrm{~nm}$ silver film thickness is sufficient to prevent direct light transmission through the silver film and coupling between SPP modes on the top and bottom surfaces of the $\mathrm{Ag}$ film. The slit width is $50 \mathrm{~nm}$, which is smaller than $\lambda / 8$ for

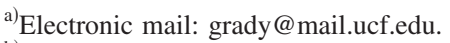

b) Also at Physics Department, University of Central Florida.
}

all of the frequencies considered here. The small slit width limits the total transmitted power and reduces the relative contribution of radiation from the slit aperture, while the sharp corners of the slit provide the local inhomogeneity required to excite bound SPP modes. The structure is illuminated from the bottom (the air and Ag interface) in the positive $z$ direction. Open boundaries are placed at $z=-60 \mathrm{~nm}$ and $z=700 \mathrm{~nm}$. In the $x$ direction, electric wall $\left(E_{y}=E_{z}=0\right)$ boundary conditions are imposed at $x=0$ and at the edge of the simulation volume at $x=L / 2$, where $L$ is a variable length of the simulation volume selected to ensure that the slow and fast modes are calculated accurately. To resolve the short wavelength and short propagation length slow modes, the grid spacing in the $x$ direction is set to $\Delta x=0.5 \mathrm{~nm}$ and $L=3000 \mathrm{~nm}$. In contrast, the fast modes have a longer wavelength and much longer propagation length, enabling the use of $\Delta x=30 \mathrm{~nm}$ and $L=80 \mu \mathrm{m}$. In both cases, the mesh density in the $z$ direction is increased near the air-silicon and silicon-silver interfaces. In the $y$ direction, magnetic wall $\left(H_{x}=H_{z}=0\right)$ boundary conditions are placed at $y= \pm 0.5 \mathrm{~nm}$. The applied boundary conditions make this effectively a twodimensional simulation of an infinite array of slits under transverse magnetic excitation; however, the simulation width $L$ was chosen to be sufficiently large to prevent interference between SPPs excited at adjacent slits.

Figure 1(a) shows the magnitude of the electric field component parallel to the layer interfaces, $E_{x}$, at a fixed phase when the slit is excited by a plane wave source with frequency $f=5.44 \times 10^{14} \mathrm{~Hz}$, corresponding to a free space wavelength of $\lambda_{f}=551 \mathrm{~nm}$. Several important features are immediately apparent. First, radiation from the slit aperture is observed, as indicated in Fig. 1(a). Second, confined SPP fields with a wavelength of approximately $500 \mathrm{~nm}$ are observed near the surface of the silver film. Finally, in the vicinity of the slit aperture, an extremely localized periodic field pattern is observed. This nanoscale optical mode is the slow SPP, shown enlarged in Fig. 1(b). The simultaneous excitation of both the slow and fast modes is possible due to the fact that the sharp edge of the slit aperture introduces a broad angular spectrum of scattered fields. This assertion was confirmed numerically in simulations in which the top edges of the slit were rounded off ${ }^{13}$ (data not shown). Note 

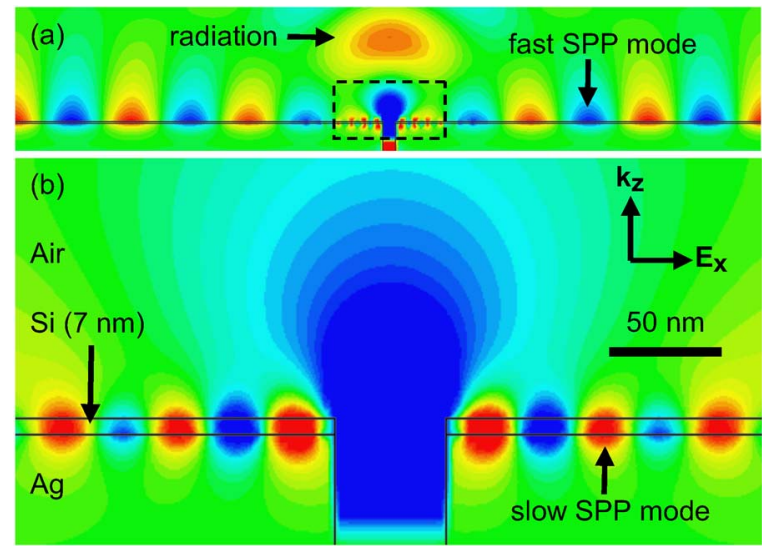

FIG. 1. (Color online) (a) Frequency domain simulation results for the $E_{x}$ field calculated at $f=5.44 \times 10^{14} \mathrm{~Hz}$. Long range, fast SPPs and radiation from the slit are visible. (b) Enlargement of the region near the slit showing the simultaneous excitation of short range, slow SPPs.

that the slow mode can be clearly distinguished despite the fact that it is superimposed on the fast mode. At this excitation frequency, the slow mode has a lateral spatial frequency $k_{x}$ of $1.21 \times 10^{8} \mathrm{~m}^{-1}$, equivalent to $\lambda_{f} / 11$. Also, it should be noted that the highly confined slow SPP fields extend beyond the Si layer into the air region, with the fields decaying to $1 / e$ in intensity within $25 \mathrm{~nm}$ of the silicon surface. This may be a sufficient distance to experimentally probe the slow mode fields and to potentially use the slow SPP fields in near-field applications.

Figure 2 shows the dispersion relation for SPPs on an infinitely thick silver layer covered with a $7 \mathrm{~nm}$ thick $\mathrm{Si}$ layer in air, calculated using the dispersion function of a three layer slab. ${ }^{14}$ For comparison, the dispersion relations of SPPs in the limit of infinitely thick ( $\mathrm{Si}-\mathrm{Ag}$ ) and infinitely thin (air-Ag) Si cover layers are included. These curves were obtained using the standard two layer SPP dispersion relation. The allowed SPP modes in this high-dielectric-contrast SPP system are largely bounded by these two limiting cases. At a free space wavelength of $595 \mathrm{~nm}$, the real part of $k_{x}$ of

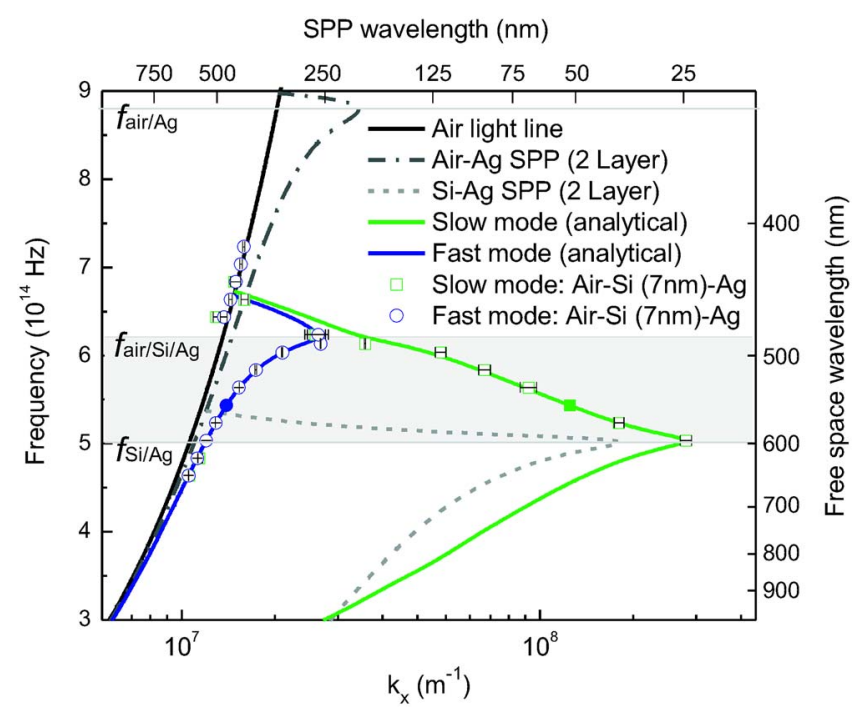

FIG. 2. (Color online) Frequency domain calculation of the dispersion curve for an air-silicon (7 nm)-silver system (symbols). Theoretical results for the fast SPP, slow SPP, Si-Ag SPP, and air-Ag SPP are presented for comparison. The frequency range in which a negative group velocity can be observed is highlighted.
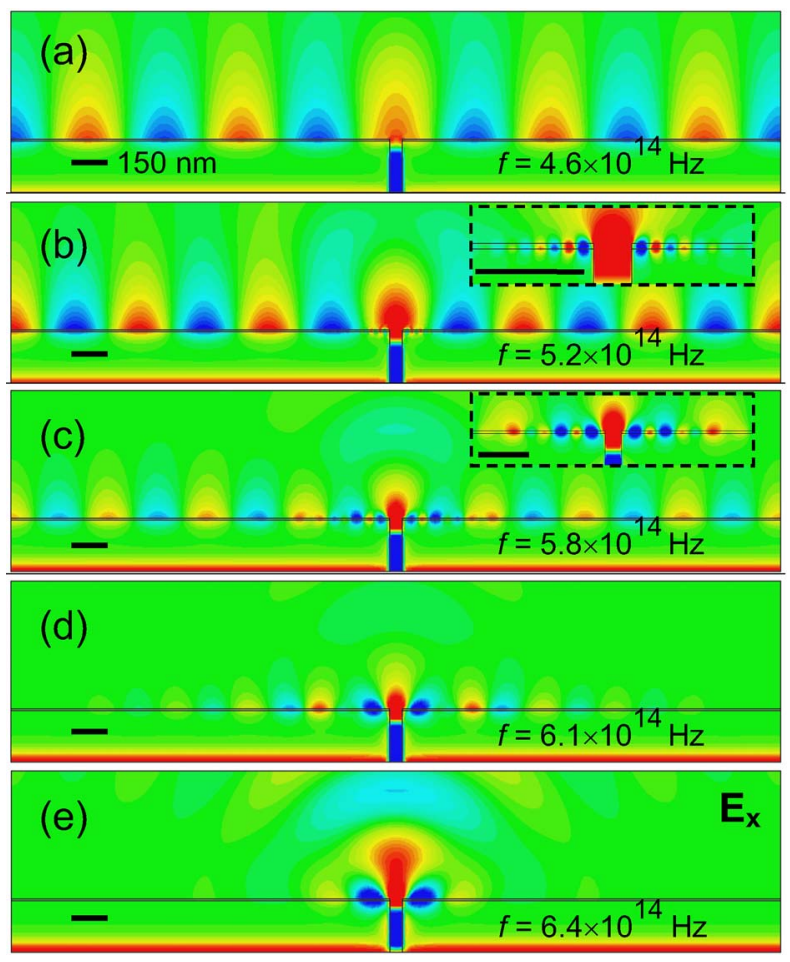

FIG. 3. (Color online) $E_{x}$ field snapshots at different frequencies and constant phase. The length of the scale bars is $150 \mathrm{~nm}$. The insets in (b) and (c) show the slow mode fields near the slit apertures.

the slow SPP reaches a maximum value of $2.55 \times 10^{8} \mathrm{~m}^{-1}$. This corresponds to a SPP wavelength as small as $25 \mathrm{~nm}$, which is equivalent to $\lambda_{f} / 24$. Interestingly, this value exceeds the maximum real $k_{x}$ allowed in the Ag-Si two layer case. $\lambda_{f} / 24$, exceeds the maximum real $k_{x}$ allowed in the high index two layer case. The filled data points in Fig. 2 represent the modes obtained under the excitation frequency used in the simulation shown in Fig. 1.

Figure 3 shows a succession of electric field patterns obtained from simulations similar to that shown in Fig. 1(a) for frequencies from $4.64 \times 10^{14}$ to $6.44 \times 10^{14} \mathrm{~Hz}$. At the lowest frequency shown, $f=4.64 \times 10^{14} \mathrm{~Hz}$, in Fig. 3(a), only the long wavelength, fast SPP is observed. In Fig. 3(b), for $f=5.24 \times 10^{14} \mathrm{~Hz}$, the fast mode is again observed. In addition, near the slit aperture, a high-spatial-frequency field pattern is observed, which is associated with the slow mode (see inset). Due to large propagation losses, the slow mode is confined to a region very close to the slit aperture. At this frequency, the wavelength of the slow mode is found to be $37.8 \mathrm{~nm}$, equivalent to $\sim \lambda_{f} / 15$. As the frequency is increased to $f=5.84 \times 10^{14} \mathrm{~Hz}$ [Fig. 3(c)], the wavelength of the fast mode is seen to decrease, as expected for light in a normally dispersive medium and for SPPs in a two layer system. In contrast, the slow SPP wavelength is seen to increase as the frequency is increased, and its spatial extent in the $z$ direction increases as well. This behavior is indicative of the anomalous dispersion of the slow plasmon branch and is associated with negative group velocity (or more accurately, opposite group and phase velocities). At a frequency of $f=6.14 \times 10^{14} \mathrm{~Hz}$ [Fig. 3(d)], the phase mismatch between the fast and slow modes has nearly disappeared and a localized field distribution is observed, corresponding to the theoretical zero group velocity point observed in Fig. 2. Finally, when the frequency is increased to $f=6.44 \times 10^{14} \mathrm{~Hz}$, 


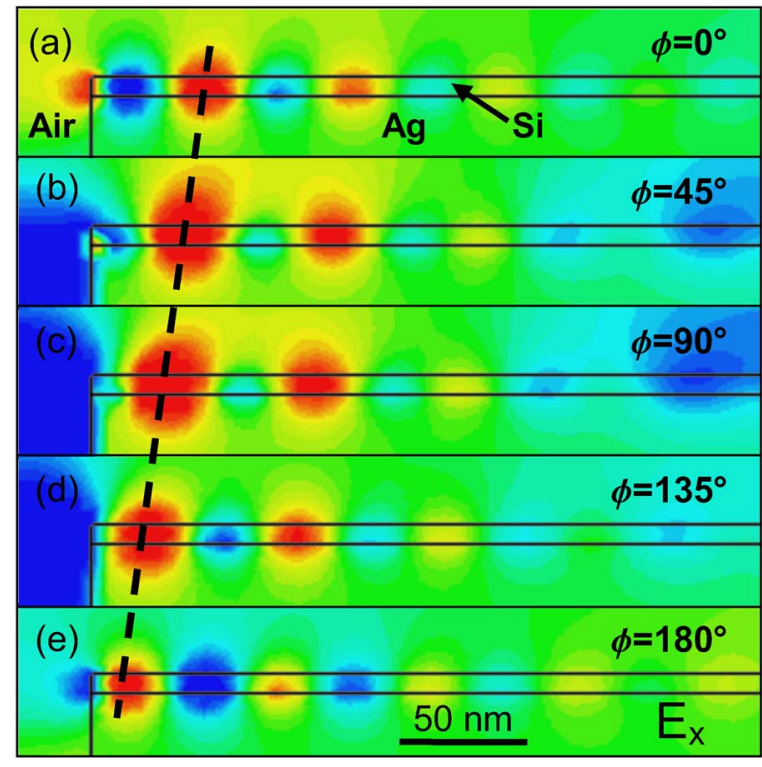

FIG. 4. (Color online) $E_{x}$ component of the slow SPP for $f=5.44$ $\times 10^{14} \mathrm{~Hz}$ with the phase varied from $0^{\circ}$ to $180^{\circ}$. As the phase $(\phi)$ is increased, the slow SPP wavefronts propagate toward the slit.

no SPPs are allowed and the dispersion curve is dominated by radiation from the slit. The wavevectors observed in these and similar simulations have been included in Fig. 2 (symbols). The results closely match the theoretical predictions, demonstrating the accuracy of the numerical methods employed.

Figure 4 depicts the real part of the $E_{x}$ field component at $f=5.44 \times 10^{14} \mathrm{~Hz}$, corresponding to the frequency used in the simulation shown in Fig. 1 , as the phase is varied from $0^{\circ}$ to $180^{\circ}$. As the phase is increased, the slow mode wavefronts move toward the slit, i.e., toward the excitation source. In contrast, for larger simulation volumes (not shown), the fast mode is observed to propagate away from slit as the phase is increased. This implies that the phase velocity of the slow mode is opposite to the direction of energy flow, which is directed away from the slit source. This negative phase velocity is a direct result of the anomalous dispersion of the slow mode: as can be seen in Fig. 2, the slow SPP branch of the dispersion curve exhibits a negative slope in the frequency band between the $\mathrm{Si}-\mathrm{Ag} \mathrm{SPP}$ resonance frequency (labeled $f_{\mathrm{Si} / \mathrm{Ag}}$ ), and the equivalent air-Si-Ag SPP resonance frequency of the fast mode (labeled $f_{\text {air } / \mathrm{S} / \mathrm{Ag}}$ ), indicative of a negative group velocity for positive $k$ values and positive phase velocity. ${ }^{15}$ The region of negative group velocity has been indicated (gray shading).
These simulations demonstrate that the experimental observation of optically excited slow SPPs will be challenging in this high dielectric contrast system due to the extremely short propagation lengths and the strong confinement of the slow mode, the presence of a background signal from the light radiated from the slit, and the significant field amplitude of the simultaneously excited fast SPP mode. Additionally, actual fabricated apertures will not have mathematically sharp corners, as used in the simulation, leading to an anticipated reduced excitation efficiency of the high-wavevector slow modes. Possible solutions may include using lower frequencies, utilizing different materials, or modifying the excitation geometry.

We have shown how a subwavelength slit may serve to excite both fast and slow SPPs on a simple layered sample that includes realistic material losses. The simulations demonstrate a minimum slow mode wavelength of $\lambda_{\text {slow }}$ $\approx \lambda_{f} / 24$ for a $7 \mathrm{~nm}$ layer of silicon on silver surrounded by air. The presence of SPP modes with negative phase velocity is demonstrated. The ability to generate SPPs with extreme ultraviolet wavelengths at surfaces using visible light could have implications in near-field microscopy, optical data storage, and optical lithography.

This work was supported in part by the Partnership project program of the Florida Photonics Center of Excellence (FPCE) and by the National Science Foundation (CAREER Award No. ECCS-0644228).

${ }^{1}$ J. Lambe and S. L. McCarthy, Phys. Rev. Lett. 37, 923 (1976).

${ }^{2}$ J. B. D. Soole and C. D. Ager, J. Appl. Phys. 65, 1133 (1989).

${ }^{3}$ M. P. Connolly, M. A. B. Whitaker, and P. Dawson, Appl. Phys. Lett. 61, 2776 (1992).

${ }^{4}$ M. P. Connolly and P. Dawson, J. Appl. Phys. 78, 5522 (1995).

${ }^{5}$ P. D. Sparks and J. E. Rutledge, Phys. Rev. B 40, 7574 (1989).

${ }^{6}$ P. D. Sparks, T. Sjodin, B. W. Reed, and J. Stege, Phys. Rev. Lett. 68 , 2668 (1992).

${ }^{7}$ A. Karalis, E. Lidorikis, M. Ibanescu, J. D. Joannopoulos, and M. Soljacic, Phys. Rev. Lett. 95, 063901 (2005).

${ }^{8}$ M. I. Stockman, Nano Lett. 6, 2604 (2006).

${ }^{9}$ MICROWAVE STUDIO is a registered trademark of CST GmbH, Darmstadt, Germany.

${ }^{10}$ P. B. Johnson and R. W. Christy, Phys. Rev. B 6, 4370 (1972).

${ }^{11}$ C. M. Herzinger, B. Johs, W. A. McGahan, J. A. Woollam, and W. Paulson, J. Appl. Phys. 83, 3323 (1998).

${ }^{12}$ G. Webb-Wood and P. G. Kik (unpublished).

${ }^{13}$ The top edges of the slit were rounded off with a radius of curvature in the range of $25-75 \mathrm{~nm}$ and conformally coated with a $7 \mathrm{~nm}$ Si layer. A clear reduction of the slow plasmon amplitude relative to that of the fast mode was observed with increasing radius of curvature.

${ }^{14}$ J. J. Burke, G. I. Stegeman, and T. Tamir, Phys. Rev. B 33, 5186 (1986).

${ }^{15}$ S. A. Maier, P. G. Kik, and H. A. Atwater, Phys. Rev. B 67, 205402 (2003). 